\title{
Litiasis renal bilateral y catéter doble J calcificado grado V: acceso percutáneo en posición supina. A propósito de un caso
}

\section{Bilateral kidney stones and grade V calcified double-J catheter: A case in which the percutaneous approach in the supine position was utilized}

\author{
Alberto Jorge Camacho-Castro, ${ }^{1 *}$ María Conchita Inzunza-Aguilar, ${ }^{2}$ Lluvia Sujey Aceves-Tello, ${ }^{3}$ Jorge Armando \\ Ramírez-Hermosillo, ${ }^{3}$ Miguel Ángel López-Chávez, ${ }^{4}$ Víctor Publio Hernández-Félix ${ }^{4}$
}

\begin{abstract}
Resumen
ANTECEDENTES: Debido al progreso en el equipo y las técnicas quirúrgicas de endourología en las pasadas dos décadas, la mayor parte de las cirugías por litiasis se han efectuado mediante accesos de mínima invasión, incluso se han ampliado las opciones de tratamiento de las complicaciones derivadas del uso de material externo, como los catéteres doble J.

CASO CLínICO: Paciente femenina de 35 años, con dolor tipo cólico en la fosa renal izquierda, con irradiación al flanco ipsilateral, fiebre no cuantificada, disuria y hematuria. En la exploración se encontró litiasis renal bilateral con hidronefrosis izquierda y se colocó un catéter doble J izquierdo. Un año después, el catéter se calcificó en su totalidad. Se realizó nefrolitotomía percutánea bilateral, litotripsia de la J distal y extracción del catéter calcificado grado V. La evolución de la paciente transcurrió sin complicaciones. Ambas sondas de nefrostomía se retiraron a los 7 días posteriores del procedimiento y el catéter doble J izquierdo 15 días después. La tomografía de control confirmó la tasa libre de litiasis. Actualmente se encuentra asintomática y con adecuada mecánica miccional.

CONCLUSIONES La nefrolitotomía percutánea en posición supina ofrece ventajas importantes para el paciente y el cirujano. El acceso y la decisión de la posición en que se practicará la punción renal debe individualizarse en cada caso.

PALABRAS CLAVE: Nefrolitotomía percutánea; catéter doble J calcificado; posición de Valdivia.
\end{abstract}

\section{Abstract}

BACKGROUND: Due to advances made in the equipment and surgical techniques of endourology over the last two decades, the majority of surgeries for treating stone disease are minimally invasive procedures. In addition, a wide range of options for the management of complications derived from the use of external material, such as double-J catheters, is now available.

CLINICAL CASE: A 35-year-old woman presented with colicky pain in the left renal fossa that radiated into the ipsilateral flank, as well as unmeasured fever, dysuria, and hematuria. The evaluation protocol revealed bilateral kidney stones with left hydronephrosis, for which a double-J catheter was placed. One year later, the catheter was completely calcified. Endoscopic bilateral percutaneous nephrolithotomy and lithotripsy at the distal portion of the catheter were performed with no incidents. Both nephrostomy catheters were removed 1 week after the procedure and the double-J catheter was removed 15 days later. Control tomography confirmed the stone-free rate. The patient is currently asymptomatic, with adequate micturition.

CONCLUSIONS: Percutaneous nephrolithotomy in the supine position offers important advantages to both the patient and the surgeon. The choice of approach and the position utilized for kidney puncture should be individualized for each case.

KEYWORDS: Percutaneous nephrolithotomy; Calcified double-J catheter; Valdivia position.

\footnotetext{
Adscrito al servicio de Urología.

2 Interno de pregrado.

${ }^{3}$ Licenciado en Enfermería quirúrgica.

${ }^{4}$ Adscrito al servicio de Anestesiología.
}

Hospital General de Culiacán Dr. Bernardo J. Gastélum (SSA).

Recibido: octubre 2017

Aceptado: julio 2018

Correspondencia

Alberto Jorge Camacho Castro

ajcc7@hotmail.com

Este artículo debe citarse como Camacho-Castro AC, Inzunza-Aguilar MC, Aceves-Tello LS, Ramírez-Hermosillo JA, López-Chávez MA, HernándezFélix VP. Litiasis renal bilateral y catéter doble J calcificado grado V: acceso percutáneo en posición supina. A propósito de un caso. Rev Mex Urol. 2018 julio-agosto;78(4):310-314. DOI: https://doi.org/10.24245/revmexurol.v78i4.1773 


\section{ANTECEDENTES}

La nefrolitotomía percutánea representa el tratamiento de elección en pacientes con cálculos renales mayores de $2 \mathrm{~cm}$ y densidad de 900 unidades Hounsfield (UH). Desde el primer reporte del tratamiento de la litiasis por vía urinaria, efectuado por Fernström y Johansonn en 1976, se han realizado múltiples avances en el equipo y las técnicas de quirúrgicas. ${ }^{1}$

La modificación en la posición del paciente, de prono a supino, reportada por primera vez por Valdivia-Uria y su grupo en 1998, mejoró la situación cardiopulmonar en el transoperatorio y con ello se abrieron nuevas posibilidades para acceder por diferentes vías (endoscópica y percutánea en un solo tiempo), para de esta manera mejorar los resultados y ofrecer una curación y recuperación más rápida de casos complejos. ${ }^{2,3}$

Ambas posiciones tienen ventajas y desventajas; por ejemplo, la posición prona proporciona un área de acción más amplia para la punción y el manejo del equipo; sin embargo, es necesario rotar al paciente y al mismo tiempo existe riesgo de producir alguna lesión en los nervios, cuello, nariz y extremidades; esta posición puede generar problemas en pacientes con enfermedad cardiovascular severa u obesidad mórbida, además de originar mayor exposición a la radiación para el cirujano y se requiere personal adicional para el cambio de posición del paciente. En sujetos con espondilitis anquilosante está contraindicada la posición en prono. ${ }^{4,5}$

En cuanto a la posición supina, el riñón se encuentra en una región anatómica que requiere menos movilidad, por lo que la punción y dilatación son más sencillas de realizar. La fluoroscopia es menos demandante en esta posición, genera menor exposición para el cirujano y, debido al fenómeno gravitatorio, la resolución libre de cálculos residuales es mayor.
La incrustación representa una complicación clínica en pacientes a quienes se colocan catéteres de derivación urinaria internos y externos; los constituyentes químicos de la orina se combinan con el catéter para formar una matriz, donde posteriormente se forman los cálculos. En marzo de 2009 se publicó un sistema de clasificación para el catéter doble J olvidado, incrustado o calcificado, FECal Ureteral Stent Grading System (Figura 1), creado por el departamento de Urología del Centro Médico de la Universidad de Loyola, Maywood, Illinois, Estados Unidos, así como un protocolo de tratamiento que permite resolver los casos con métodos más efectivos, según el grado de calcificación del catéter. ${ }^{6}$

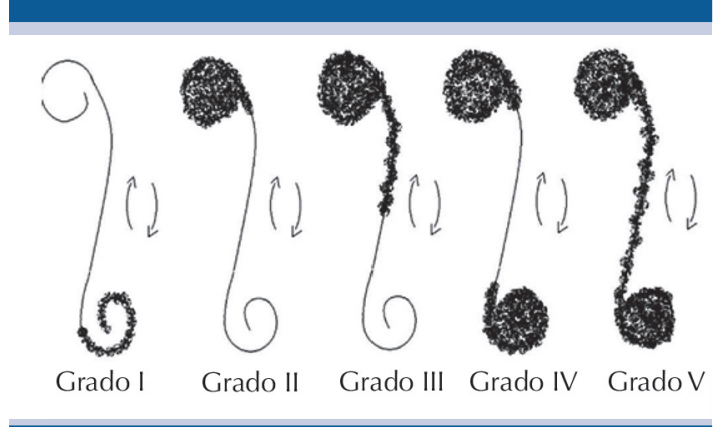

Figura 1. Clasificación FECal para catéteres doble J.

\section{CASO CLÍNICO}

Paciente femenina de 35 años de edad, con dolor tipo cólico en la fosa renal izquierda e irradiación al flanco ipsilateral, concomitante con fiebre no cuantificada, síntomas urinarios y hematuria macroscópica, sin formación de coágulos. En la exploración se encontró litiasis renal bilateral de gran tamaño, cuyo tratamiento inicial consistió en la colocación de un catéter doble J izquierdo. Durante el seguimiento tuvo fractura de cadera izquierda; se le colocó una prótesis y un año después se observó la calcificación total del catéter doble J (Figura 2), por lo 


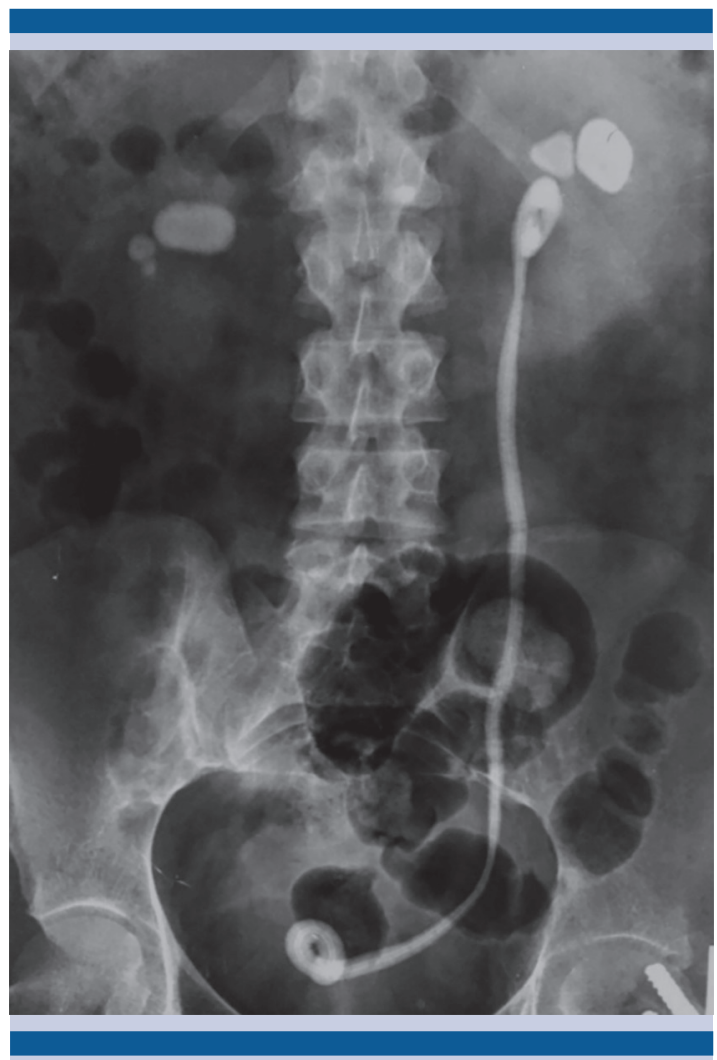

Figura 2. Estudio de imagen preoperatorio.

que ingresó al servicio de Urología del Hospital General de Culiacán Dr. Bernardo J. Gastélum para valoración y tratamiento.

La tomografía computada reportó litiasis renal derecha, de aproximadamente $3.5 \times 2.5 \mathrm{~cm}$, con
1080 UH, e izquierda de $3 \times 1.5 \mathrm{~cm}$, con 1115 UH y catéter doble J calcificado en su totalidad. Se decidió el acceso percutáneo, en posición de Valdivia-Galdakao. El procedimiento se Ilevó a cabo en dos tiempos: en el primero se realizó nefrolitotomía percutánea derecha, con duración de 60 minutos y sangrado estimado de 100 cc, y en el segundo se efectuó litotripsia, con un litotriptor neumático. Al cabo de un mes de la intervención quirúrgica se practicó nefrolitotomía percutánea izquierda, con acceso endoscópico para litotripsia de la J distal y extracción del catéter doble J calcificado grado $\mathrm{V}$ (Figura 3). El procedimiento tuvo duración de 160 minutos y sangrado estimado de 150 cc. La fragmentación de ambos rizos del catéter y del cálculo renal total se llevó a cabo con un litotriptor neumático; se extrajo el catéter en su totalidad (Figura 4). En ambos procedimientos se puncionó en el cáliz inferior y no se registraron incidentes.

La evolución de la paciente transcurrió sin complicaciones (Figura 5). Ambas sondas de nefrostomía se retiraron una semana después del procedimiento y el catéter doble J izquierdo 15 días posteriores. La tomografía de control confirmó la tasa libre de litiasis. Actualmente se encuentra asintomática y con adecuada mecánica miccional.

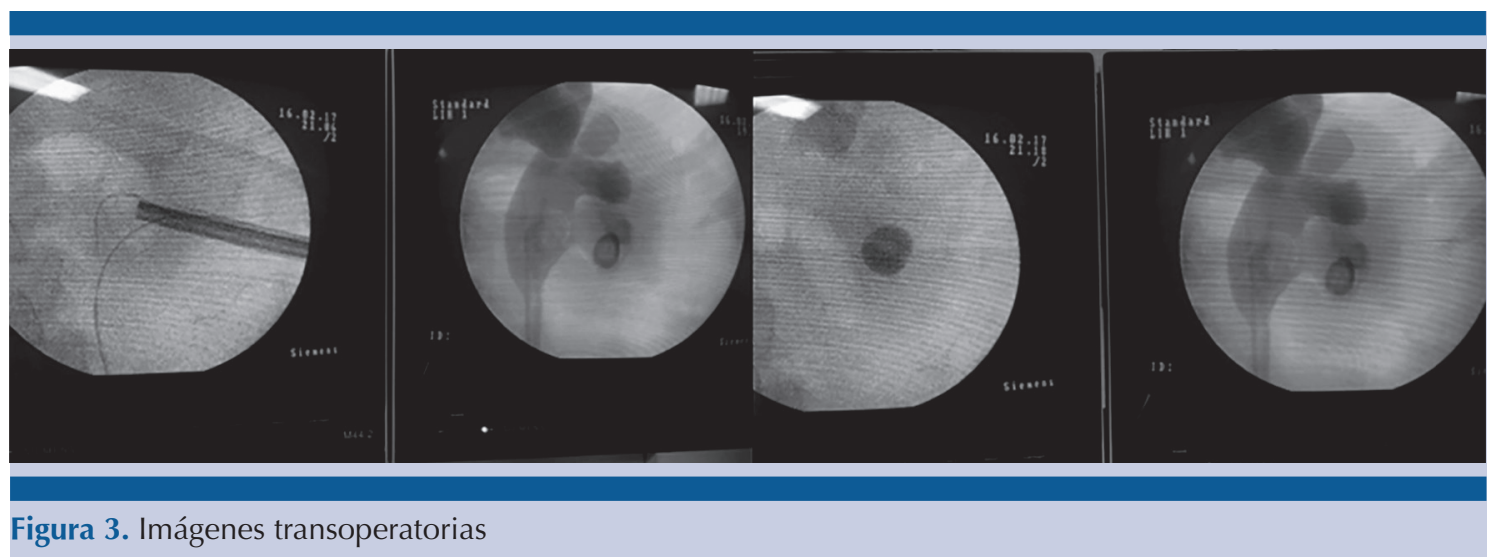




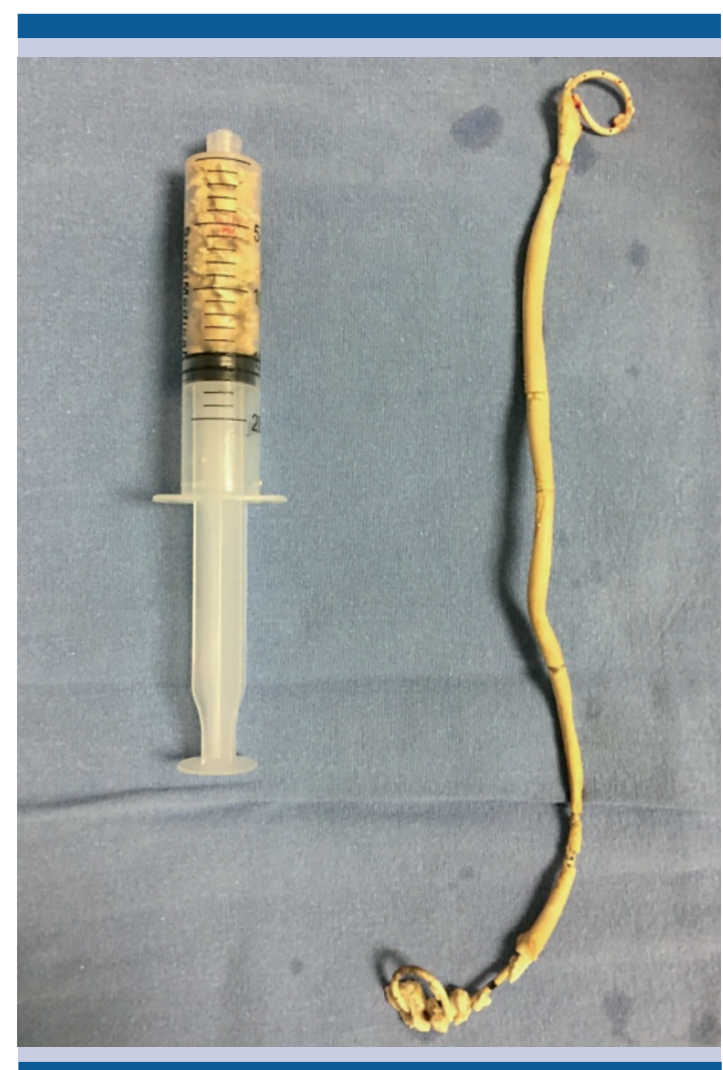

Figura 4. Catéter doble J calcificado grado V.

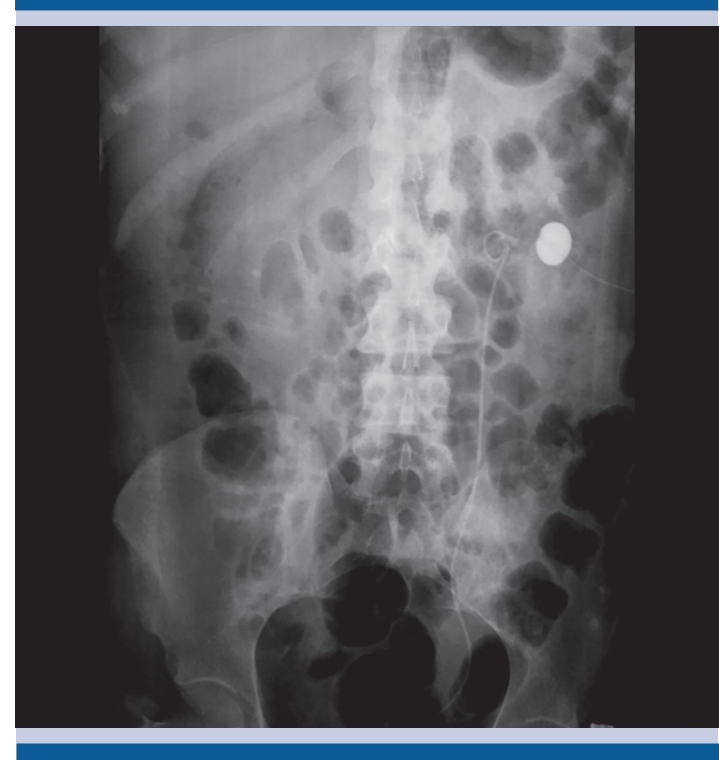

Figura 5. Imagen posoperatoria sin complicaciones.

\section{DISCUSIÓN}

Aunque la nefrolitotomía percutánea se considera el tratamiento de elección en pacientes con cálculos de gran tamaño (mayores de $2 \mathrm{~cm}$ ), no se encuentra exenta de complicaciones, incluso puede elevar los días de hospitalización y costos médicos. Conforme aumenta el progreso en procedimientos de mínima invasión, al mismo tiempo evolucionan los equipos y las técnicas de acceso con diversos resultados.

De acuerdo con un estudio de la Sociedad de Endourología, se ha reportado mayor tasa libre de litiasis en posición prona; sin embargo, hasta el momento no existe un consenso que sugiera que esta posición ofrezca mejores resultados, por lo que debe personalizarse el abordaje en cada caso. ${ }^{8}$

Diversas investigaciones reportan resultados similares al comparar la posición prona versus supina en relación con la tasa libre de litiasis, cuyo promedio es de $81 \%$ (62-95\%). Aunque muchas veces se ha considerado la carga litiásica como el factor predictivo más importante en la extracción de cálculos renales, existen factores adicionales que influyen de manera significativa en los resultados como: tratamiento previo para litiasis, detección de cálculos coraliformes, localización y cantidad total de cálculos.

Algunos estudios prospectivos que compararon la nefrolitotomía percutánea unilateral versus bilateral simultánea señalaron una tasa de éxito de $96 \%$ en la primera, con requerimiento de reintervención en $2.8 \%$ de los pacientes; sin embargo, la comparación final reportó que la tasa libre de cálculo residual, insuficiencia renal posterior al procedimiento, estancia hospitalaria, indicación de analgésicos y complicaciones no mostraron diferencias significativas entre ambas modalidades de tratamiento. ${ }^{9,10}$ 
La extracción de catéteres doble J retenidos e incrustados representa un reto quirúrgico para el urólogo e incrementa el riesgo de morbilidad del paciente; sin embargo, existen diversas opciones de tratamiento en estos casos, como la cirugía abierta, laparoscópica, percutánea o endoscópica con litotricia (hidráulica y láser). En la paciente de este estudio la posición supina permitió el acceso mixto y resolver la complicación del catéter doble J, con resultados y evolución satisfactorios.

\section{CONCLUSIONES}

La nefrolitotomía percutánea en posición supina ofrece ventajas importantes para el paciente y el cirujano; sin embargo, el acceso y la decisión de la posición en que se practicará la punción renal debe individualizarse en cada caso.

\section{REFERENCIAS}

1. Quaresima L, et al. Safety and efficacy of percutaneous nephrolithotomy in the Galdakao-Modifiec Supine Val- divia position: A prospective analysis. DOI: https://doi. org/10.4081/aiua.2016.2.93

2. Falahatkar S, Mokhtari G, Teimoori M. An update in supine versus prone percutaneous nephrolithotomy: a metaanalysis. Urol J 2016;13(5):2814-2822.

3. Zapata-González J, Camacho-Castro J, García S. Nefrolitotomía percutánea en posición supina: nuestra experiencia. Rev Mex Urol 2014;74(6):336-341.

4. Heinze-Rodríguez A, et al. Manejo de litiasis renal con nefrolitotomía percutánea: Experiencia en un hospital de referencia. Rev Mex Urol 2014;74(4):211-215.

5. Barradas-Huervo $E$, et al. Experiencia en nefrolitotomía percutánea en el Centro Médico Nacional "20 de Noviembre". Rev Mex Urol 2008;68(2):69-87

6. Camacho-Castro AJ, et al. Manejo de catéteres doble J calcificados en el Hospital General "Dr. Manuel Gea González". Rev Mex Urol 2013;73(4):155-159.

7. Castillo $\mathrm{O}$, et al. Cirugía percutánea de la litiasis coraliforme. Rev Chil Cir 2008;60(5):393-397.

8. Yu J, et al. Risk factors for pulmonary complications after percutaneous nephrolithotomy. Medicine 2016;95:35.

9. Skolarikos A, et al. Percutaneous Nephrolithotomy and its Legacy. DOI: https://doi.org/10.1016/j.eururo.2004.08.009

10. Karalar $\mathrm{M}$, et al. Effects of parenchymal thickness and stone density values on percutaneous nephrolithotomy outcomes. DOI: 10.12659/MSM.898212

11. Michel MS, et al. Complications in percutaneous nephrolithotomy. DOI: https://doi.org/10.1016/j.eururo.2006.10.020 